\title{
A Linear Quadratic Gaussian Framework for Optimal Networked Control System Design ${ }^{\dagger}$
}

\author{
Mohammad Tabbara, Dragan Nešić and Nuno C Martins
}

\begin{abstract}
The purpose of this paper is to develop an optimal controller design framework for a class of linear time invariant (LTI) networked control systems with stochastic intertransmission intervals and random scheduling protocols perturbed by stochastic noise processes. Whereas the authors' previous works examine sufficient conditions that lead to robust $\left(L_{p}\right.$, ISS) notions of stability ex post facto controller design ignoring the network, this paper examines controller design taking the network into account in an appropriate sense. In particular, we assume zero-order hold between transmission instants and our optimality criteria apply to the discrete-time system induced by examining the NCS only at transmission instants. We do, however, develop performance bounds between such instants (where no claim of optimality is made).
\end{abstract}

\section{INTRODUCTION}

Advances in both communication technology and research in control theory open up the opportunity for applications involving increasingly large and complex control systems, including systems consisting of physically disparate components spread across large distances and systems with imperfect communication. These systems lend themselves naturally to the use of an appropriate network for communication amongst system components together with a scheduling protocol within the context of a networked control system (NCS). While an NCS approach to control system implementation offers significant engineering advantages, including lower cost, weight and standards-based components, there are challenges presented in the synthesis of control laws and stability and robustness analysis.

An emulation-based approach to NCS design calls for first designing a continuous-time dynamical feedback controller ignoring the network, selecting a scheduling protocol from a suitable class of protocols and then increasing the transmission rate sufficiently to ensure that a notion of (robust) stability is achieved. Several such classes of scheduling protocols were introduced and associated transmission rate bounds were developed in [1]-[4] for various notions of deterministic and stochastic ( $L_{p}$ and $\mathcal{K} \mathcal{L}+$ sup) inputto-state, input-output stability, uniform global and uniform exponential stability.

$†$ This work was supported by the Australian Research Council under the Australian Professional Fellowship \& Discovery Grants Scheme. Nuno C. Martins was funded by Award NSF EECS 0644764 CAREER: Distributed control of dynamic systems using a wireless communcation medium: two new paradigms.

M. Tabbara and D. Nešić are with the Department of Electrical and Electronic Engineering, The University of Melbourne, Parkville, VIC 3010 Australia m.tabbaradee.unimelb.edu. au

N.C. Martins is with the Department of Electrical and Computer Engineering, University of Maryland, College Park, MD 20740, USA nmartinseisr.umd.edu
Away from scheduling, several papers have examined the data rate limitations for stabilizability over finite capacity data channels. For linear systems, [5], [6] have characterized the minimum data rate required for stability together with stabilizing feedback control laws achieving the data rate bounds.

This paper aims to develop a systematic synthesis framework for NCS away from the boundaries of fundamental data rate constraints, in a similar vein to the aforementioned emulation-based approach. The framework is similar in spirit to that in [7] with the key differences being that we develop our results in a stochastic setting, do not assume any parametrization, or, indeed, linearity of the control and our scheduling protocol is fixed. Restricting our attention to linear continuous-time systems perturbed by Gaussian noise (so-called diffusion processes), we provide an optimal feedback control law synthesis framework for the family of Ethernet-like protocols. To that end, we first develop a characterization of Ethernet as a protocol as used in a control network implemented over an imperfect communication channel (non-zero probability of data-loss); design a finitehorizon feedback control law assuming only actuator values are transmitted across the network; develop necessary and sufficient conditions as functions of the data-loss probability and average data-rate for existence of a stationary infinitehorizon feedback control that is the limit of the sequence of finite-horizon control laws and apply the design framework to a case study.

\section{ETHERNET-LIKE SCHEDULING}

Ethernet (IEEE 802.11) and its wireless counterparts are the de facto standard for computer networks across a broad range of applications and we describe a simplified version of the protocol under NCS traffic assumptions in this section. We assume that:

1) Sensors or data sinks "fast sample" in continuous-time, hence, sinks will always transmit if the channel is deemed to be idle;

2) the network is a broadcast channel, that is, (successful) data transmissions are received by every data sink on the network;

3) the network-traffic is not mixed and is solely due to the transmission of sensor and actuator data;

4) the multiple nodes transmitting simultaneously will result in a collision and complete data loss; and

5) the network channel is non-ideal, admitting the possibility of data losses once transmission has commenced. 
The data loss is complete and the loss process is Bernoulli with the probability of a loss given by $p_{0} \geq 0$.

Together with these assumptions, each source $i \in[1, \ell]$ can be seen as adopting Algorithm 1 (see [8, Chapter 2] for a description of the operation of Ethernet).

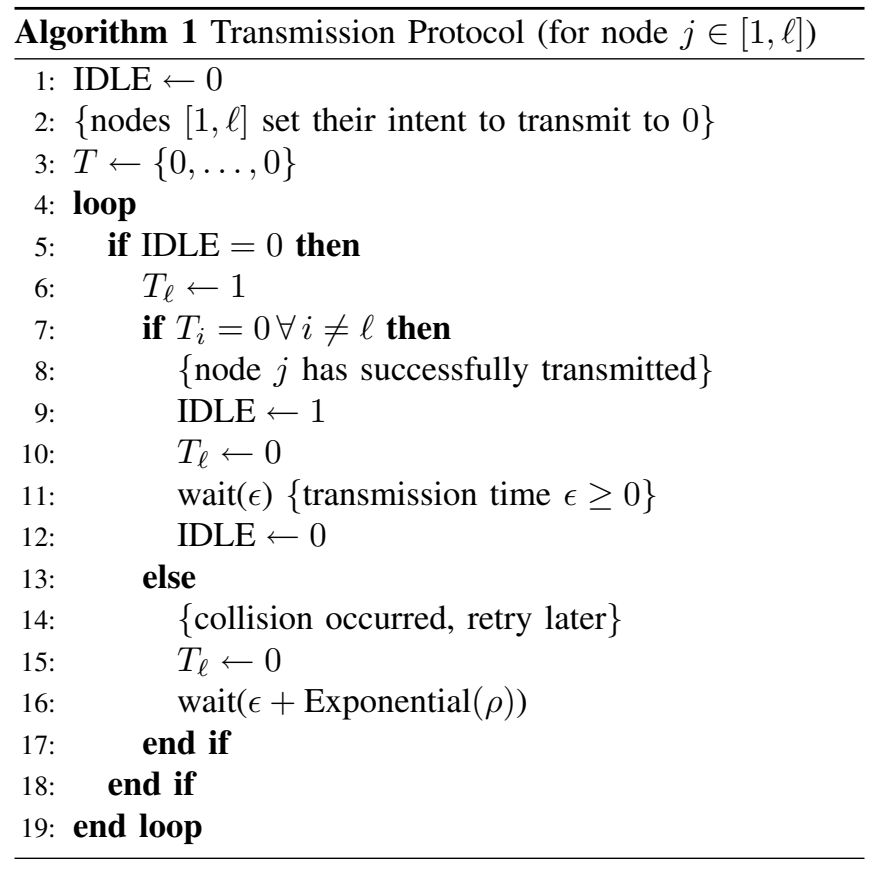

With the assumption that there is always data to transmit and that the channel is initially idle, a collision is guaranteed to occur upon initialization of the NCS.

Proof: $I D L E=0 \Rightarrow T_{i}=1$ for all $i \in[1, \ell]$ hence line 7 of Algorithm 1 is false for all $i \in[1, \ell]$.

The node transmitting next will be the node that waits the least amount of time and that time is given by

$$
s=\epsilon+\min \left\{Z_{1}, \ldots, Z_{l}\right\},
$$

where $Z_{i} \sim \operatorname{Exponential}(\rho)$ and mutually independent. Hence, $s-\epsilon \sim \operatorname{Exponential}(\lambda)$, where $\lambda=\rho \ell$. In particular, the probability that there are nodes that wait an equal amount of time after the initial collision is zero and $\mathbf{P}\left\{s-\epsilon=Z_{i}\right\}=1 / \ell$.

Hence, the probability that any source node successfully transmits is $\frac{1-p_{0}}{\ell}$ and inter-transmission intervals is given by

$$
s=\epsilon+\delta \quad \text { where, } \quad \delta \sim \operatorname{Exponential}(\lambda) .
$$

To simplify the presentation, and assuming that the transmission rate is relatively high compared to the packet (frame) size, we henceforth set $\epsilon=0$ and, thus, inter-transmission intervals are iid and $s \sim \operatorname{Exponential}(\lambda)$.

\section{Networked Control System Model}

For simplicity, we only consider the case of transmitting controls over the network:

$$
\dot{x}=F x+G u+\tilde{w},
$$

where $\tilde{w}$ is a zero-mean Gaussian noise process with covariance matrix $\tilde{\Lambda}$.

We assume a zero-order hold policy for control between transmission instants. In light of this, given some scheduling protocol $\Psi$ whose effect is to reset components of the control to newly transmitted values: $\tilde{u}^{+}=\Psi \tilde{u}+(I-\Psi) u$, we can rewrite (1) explicitly as the system

$$
\begin{aligned}
\dot{x} & =F x+G \tilde{u}+\tilde{w} \quad t \in\left[t_{i}, t_{i+1}\right] \\
\tilde{u}\left(t_{i}^{+}\right) & =\Psi \tilde{u}\left(t_{i}\right)+(I-\Psi) u\left(t_{i}\right),
\end{aligned}
$$

that is, with our zero-order hold policy, we only admit the possibility of piecewise constant control that is recomputed at each transmission instant.

Within this paper, we only examine the case of Ethernetlike scheduling, as described in Section II and first introduced in [2], thus, $\Psi$ is an iid process. In particular, $\mathbf{P}\{\Psi=I\}=$ $p_{0}$ and, assuming equally probable successful transmissions of any other source, say, node $i$,

$$
\mathbf{P}\left\{\Psi=M_{i}\right\}=\frac{1-p_{0}}{\ell},
$$

where $\ell \geq 1$ denotes the number of source nodes and where

$$
M_{i}=1-\operatorname{diag}\left\{0, \ldots, 0,1_{i i}, 0, \ldots,\right\} .
$$

Hence, examining the system only at transmission instants, we have the exact discrete-time description as an affine linear time-invariant system parameterized by the integration period $s:$

$x^{+}=\exp (F s) x+\int_{0}^{s} \exp (F \theta) G \Psi \tilde{u} d \theta+G(I-\Psi) u+w$

$\tilde{u}^{+}=\Psi \tilde{u}+(I-\Psi) u$

where $w$ is a now a time-varying (non-stationary) Gaussian noise process (a linear function of $\tilde{w}$ ) independent of $(x, u)$, $\Psi$ is the aforementioned (random) scheduling protocol and $(u, \tilde{u})$. Initially, the only assumption that we make is that the integration periods $s \geq 0$ are iid (and, hence, why it suffices to parameterize the integration by a single random variable), $\Psi$ is an iid process and independent of $s$. The variable $\tilde{u}$ plays the role of the last previously accumulated vector of controls and $u$ is the current update. By augmenting the state space, (4) can be expressed as the following LTI system:

$$
\begin{array}{r}
{\left[\begin{array}{l}
x^{+} \\
\xi^{+}
\end{array}\right]=\left[\begin{array}{cc}
\exp (F s) & \left(\int_{0}^{s} \exp (F \theta) d \theta\right) G \Psi \\
0 & \Psi
\end{array}\right]\left[\begin{array}{l}
x \\
\xi
\end{array}\right]} \\
+\left[\begin{array}{c}
G(I-\Psi) \\
I-\Psi
\end{array}\right] u+w .
\end{array}
$$

\section{Optimal Synthesis: State-FEEDBACK}

We rewrite (6) in the form

$$
z^{+}=A z+B u+w
$$

where $z=(x, \xi)$, 


$$
\begin{gathered}
A=\left[\begin{array}{cc}
\exp (F s) & \left(\int_{0}^{s} \exp (F \theta) d \theta\right) G \Psi \\
\Psi
\end{array}\right], \\
B=\left[\begin{array}{c}
G(I-\Psi) \\
I-\Psi
\end{array}\right]
\end{gathered}
$$

with the tacit understanding that $A, B$ are functions of $(s, \Psi)$. Consider the controller design problem with the finite-horizon quadratic cost:

$$
J(u)=\mathbf{E}\left\{\sum_{i=0}^{N-1}\left(z_{i}^{T} Q z_{i}+u_{i}^{T} R u_{i}\right)+z_{N}^{T} Q_{N} z_{N}\right\}
$$

in the presence of a random iid scheduling protocol (process) $\Psi$ as previously described.

By holomorphic functional calculus (see [9, Chapter VII], for instance), we may further rewrite $A$ as the following matrix

$$
A=\left[\begin{array}{cc}
\exp (F s) & F^{-1}(\exp (F s)-I) G \\
0 & \Psi
\end{array}\right] .
$$

From [10, Sec 4.1], the optimal control law is linear and given by $u_{k}=L_{k} z_{k}$, where the gain matrices $L_{k}$ are given by

$$
L_{k}=-\left(R_{k}+\mathbf{E}\left\{B_{k}^{T} K_{k+1} B_{k}\right\}\right)^{-1} \mathbf{E}\left\{B_{k}^{T} K_{k+1} A_{k}\right\},
$$

and where the matrices $K_{k}$ are given by the recursive equation

$$
\begin{gathered}
K_{N}=Q_{N}, \\
K_{k}=\mathbf{E}\left\{A_{k}^{T} K_{k+1} A_{k}\right\} \\
-\mathbf{E}\left\{A_{k}^{T} K_{k+1} B_{k}\right\} \\
\times\left(R_{k}+\mathbf{E}\left\{B_{k}^{T} K_{k+1} B_{k}\right\}\right)^{-1} \mathbf{E}\left\{B_{k}^{T} K_{k+1} A_{k}\right\}+Q .
\end{gathered}
$$

In principle, the finite-horizon optimal design problem is solved at this point but is not necessarily a stationary policy. That is, $L_{k}$ may be time-varying.

\section{A. Infinite-Horizon Design}

For an infinite-horizon design, we seek a stationary feedback strategy that is optimal for costs of the form:

$$
J(u)=\mathbf{E}\left\{z^{T} Q z+u^{T} R u\right\},
$$

that (through ergodicity) be considered as the ensemble average of finite-horizon costs as $N \rightarrow \infty$, though, as [10, Sec 4.1] establishes, (9) need not converge to yield a positive symmetric matrix $P$ as the solution. If such a solution does exist, then a stationary solution to the infinite-horizon design problem exists and is given by the control law $u=L z$, where

$$
L=-\left(\mathbf{E}\left\{B^{T} P B\right\}+R\right)^{-1} \mathbf{E}\left\{B^{T} P A\right\},
$$

and, in principle, evaluation of the convergence of (9) can be carried out numerically.

To emphasize the dependence of the system equation on the relevant iid random variables, we rewrite

$$
z^{+}=A(s, i) z+B(s, i) u+w
$$

where $s$ denotes the (exponentially-distributed) integration period for the transmission and $i$ denotes the node ${ }^{1}$ that will be transmitted - that is, fixing $i$ fixes the value of the scheduling function $\Psi$. Thus, we can regard (10) as a linear discrete-time with infinite Markov jump parameters (though in this case, the Markov process is trivial since the variables are iid). What has not been explicitly stated before and not assumed in the either the finite-horizon or infinite-horizon dynamic programming solutions to the optimal design problem is that the control strategy may depend on the integration period $s$ and the choice of node $i$. Both $s$ and $i$ are known by all nodes just prior to transmission and their knowledge can, in principle, be incorporated in the design of the optimal control strategy. We make this explicit:

Assumption 1: It is assumed that all nodes can measure the integration time (time since last transmission), $s$ and that the success of a particular node $i$ in acquiring access to the network becomes known just prior to transmission of the payload data.

Sufficient conditions for the existence of an LQ-optimal feedback control in this setting were presented in [11] but are generally unwieldy. In the sequel, we present necessary and sufficient conditions for the existence of an optimal feedback strategy under Assumption 1 when transmissions are equidistant - that is, when $s=$ constant $>0$.

Theorem 1: Suppose $A_{i} \in \mathbb{R}^{n \times n}, B_{i} \in \mathbb{R}^{n \times m}$ for $0 \leq$ $i \leq \ell$. Let $0 \leq p_{0}<1$ and let $p=\frac{1-p_{0}}{\ell}$. Consider the stochastic difference equation

$$
z^{+}=A_{\theta} z+B_{\theta} u+w
$$

where $w \in \mathbb{R}^{n}$ is a zero-mean Gaussian white noise with covariance matrix $\Lambda, \mathbf{P}\left\{\left(A_{\theta}, B_{\theta}\right)=\left(A_{i}, B_{i}\right)\right\}=p$ for $i \neq 0$ and $p_{0}$ otherwise, iid. Then for every $\gamma \geq 0$, the following two statements are equivalent:

(i) There exists a map $\mu:\{0,1, \ldots, \ell\} \times \mathbb{R}^{n} \rightarrow \mathbb{R}^{m}$ such that (11) with $u=\mu(\theta, z)$, where the random variable $\theta$ denotes the current node, has a stationary zero mean solution satisfying:

$$
\mathbf{E}|C z+D u|^{2} \leq \gamma
$$

(ii) There exist $X_{0}=\left[\begin{array}{cc}X_{z z} & 0 \\ 0 & 0\end{array}\right], X_{i}=\left[\begin{array}{cc}X_{z z} & X_{z u_{i}} \\ X_{u_{i} z} & X_{u_{i} u_{i}}\end{array}\right] \in$ $\mathbb{R}^{(n+m) \times(n+m)}, i \in\{1, \ldots, \ell\}$ with $X_{0}, X_{i} \geq 0$ such that:

$$
\begin{gathered}
X_{z z}=\Lambda+p_{0}\left[\begin{array}{ll}
A_{0} & B_{0}
\end{array}\right] X_{0}\left[\begin{array}{ll}
A_{0} & B_{0}
\end{array}\right]^{T} \\
+p \sum_{i=1}^{\ell}\left[\begin{array}{ll}
A_{i} & B_{i}
\end{array}\right] X_{i}\left[\begin{array}{ll}
A_{i} & B_{i}
\end{array}\right]^{T}, \\
\gamma \geq p_{0} \operatorname{tr}\left(\left[\begin{array}{ll}
C & D
\end{array}\right] X_{0}\left[\begin{array}{ll}
C & D
\end{array}\right]\right) \\
+p \sum_{i=1}^{\ell} \operatorname{tr}\left(\left[\begin{array}{ll}
C & D
\end{array}\right] X_{i}\left[\begin{array}{ll}
C & D
\end{array}\right]\right)
\end{gathered}
$$

Moreover, if $\left\{X_{i}\right\}_{i=0}^{\ell}$ satisfy the conditions of (ii) with the minimum feasible $\gamma$, then (11) with the linear control

\footnotetext{
1"Node" $i=0$ denotes a collision and indicates that no node was successful in transmitting data in the respective interval.
} 
law $\mu(i, z)=X_{u_{i} z} X_{z z}^{-1} z$ has a solution satisfying (12). If $\left\{X_{i}\right\}_{i=0}^{\ell}>0$, then the control law is also stabilizing.

Proof: To prove the implication (i) $\Rightarrow$ (ii), suppose that (i) holds for the map $\mu$ and the process $z$. For a fixed mode $i$, let $u_{i}=\mu(i, z)$. Define

$$
\begin{gathered}
X_{z z}=\mathbf{E}\left\{z z^{T}\right\}, \quad X_{u_{i} u_{i}}=\mathbf{E}\left\{u_{i} u_{i}^{T}\right\}, \\
X_{z u_{i}}=\mathbf{E}\left\{z u_{i}^{T}\right\}, \quad X_{u_{i} z}=X_{z u_{i}}^{T} \quad \text { and } \\
X_{0}=\left[\begin{array}{cc}
X_{z z} & 0 \\
0 & 0
\end{array}\right] \quad X_{i}=\left[\begin{array}{cc}
X_{z z} & X_{z u_{i}} \\
X_{u_{i} z} & X_{u_{i} u_{i}}
\end{array}\right] .
\end{gathered}
$$

By conditioning on the event that the current node is $\theta=i$ and using the law of total expectation, we have:

$$
\begin{aligned}
\mathbf{E}|C z+D u|^{2} & =\operatorname{tr}\left(\mathbf{E}\left\{(C z+D u)(C z+D u)^{T}\right\}\right) \\
& =\mathbf{E}\left\{\operatorname{tr}\left(\mathbf{E}\left\{(C z+D u)(C z+D u)^{T} \mid u=u_{i}\right\}\right)\right\} \\
& =\mathbf{E}\left\{\operatorname{tr}\left(\left[\begin{array}{ll}
C & D
\end{array}\right] X_{i}\left[\begin{array}{ll}
C & D
\end{array}\right]\right)\right\} \\
& =p_{0} \operatorname{tr}\left(\left[\begin{array}{ll}
C & D
\end{array}\right] X_{0}\left[\begin{array}{ll}
C & D
\end{array}\right]\right) \\
& \quad+p \sum_{i=1}^{\ell} \operatorname{tr}\left(\left[\begin{array}{ll}
C & D
\end{array}\right] X_{i}\left[\begin{array}{ll}
C & D
\end{array}\right]\right) \leq \gamma
\end{aligned}
$$

The remaining conditions of (ii) follow directly from the corresponding conditions of (i).

To prove the implication (ii) $\Rightarrow$ (i), suppose that (ii) holds. Since $\left\{X_{i}\right\}_{i=0}^{\ell} \geq 0, X_{u_{i} u_{i}} \geq X_{u_{i} z} X_{z z}^{-1} X_{z u_{i}}$ for $i \in\{1, \ell\}$ and equality can be assumed without restriction as reducing $X_{u_{i} u_{i}}$ can only reduce the stationary cost. The difference equation (11) with $u_{i}=L_{i} z$, where $L_{i}=X_{u_{i} z} X_{z z}^{-1}$ can be written

$$
z^{+}=\left(A_{\theta}+B_{\theta} L_{\theta}\right) z+w
$$

Let $z(0)$ be a Gaussian stochastic variable with

$$
\mathbf{E}\{z(0)\}=0 \quad \mathbf{E}\left\{z(0) z(0)^{T}\right\}=X_{z z} .
$$

By linearity, $z(1)$ is also Gaussian with $\mathbf{E}\{z(1)\}=0$ and

$$
\begin{aligned}
& \mathbf{E}\left\{z(1) z(1)^{T}\right\} \\
& =\mathbf{E}\left\{\mathbf{E}\left\{z(1) z(1)^{T} \mid \theta=i\right\}\right\} \\
& =\mathbf{E}\left\{\left\{\left(A_{i}+B_{i} L_{i}\right) \mathbf{E}\left\{z(0) z(0)^{T}\right\}\left(A_{i}+B_{i} L_{i}\right)^{T}\right\}+\Lambda\right. \\
& =\mathbf{E}\left\{\left(A_{i}+B_{i} L_{i}\right) X_{z z}\left(A_{i}+B_{i} L_{i}\right)^{T}\right\}+\Lambda \\
& =\Lambda+p_{0}\left[\begin{array}{ll}
A_{0} & B_{0}
\end{array}\right] X_{0}\left[\begin{array}{ll}
A_{0} & B_{0}
\end{array}\right]^{T} \\
& \quad+p \sum_{i=1}^{\ell}\left[\begin{array}{ll}
A_{i} & B_{i}
\end{array}\right] X_{i}\left[\begin{array}{ll}
A_{i} & B_{i}
\end{array}\right]^{T}=X_{z z} .
\end{aligned}
$$

Thus, $z$ is stationary and $\mathbf{E}\left\{z z^{T}\right\}=X_{z z}$. Multiplying by $\left[\begin{array}{ll}I & L_{i}^{T}\end{array}\right]$ from the right and its transpose from the left yields

$$
\mathbf{E}\left\{\left[\begin{array}{l}
z \\
u
\end{array}\right]\left[\begin{array}{l}
z \\
u
\end{array}\right]^{T} \mid \theta=i\right\}=\left[\begin{array}{c}
I \\
L_{i}
\end{array}\right] \mathbf{E}\left\{z z^{T}\right\}\left[\begin{array}{c}
I \\
L_{i}
\end{array}\right]^{T}=X_{i}
$$

where, by convention, $L_{0}=0$.

\section{Output Feedback}

We restrict our attention to the case where intertransmission intervals are fixed with $s=\tau>0$ and examine the case of output feedback where only outputs are scheduled for transmission. Previously transmitted outputs $y$ are accumulated into the state variable $\xi$ and, thus, by augmentation, the NCS is given by

$$
\begin{aligned}
{\left[\begin{array}{l}
x^{+} \\
\xi^{+}
\end{array}\right] } & =\left[\begin{array}{cc}
\exp (F s) & 0 \\
(I-\Psi) H & \Psi
\end{array}\right]\left[\begin{array}{l}
x \\
\xi
\end{array}\right]+\left[\begin{array}{c}
G \\
I
\end{array}\right] u+w \\
y & =\left[\begin{array}{cc}
(I-\Psi) H & 0 \\
0 & I
\end{array}\right]\left[\begin{array}{l}
x \\
\xi
\end{array}\right]+v
\end{aligned}
$$

where $H$ denotes the output matrix and $v$ is zero mean white Gaussian noise with covariance $\Omega$. Let $A_{\theta}, B_{\theta}, C_{\theta}$ denote the system matrices for (15)-(16).

The following result describing a Kalman-like state estimator is inspired, in part, by the presentation in [12] examining output feedback control design for distributed systems.

Theorem 2: Suppose $D_{i} \in \mathbb{R}^{n \times n}, C_{i} \in \mathbb{R}^{p \times n}$ for $0 \leq i \leq$ $\ell$. Let $0 \leq p_{0}<1$ and let $p=\frac{1-p_{0}}{\ell}$. Consider the stochastic difference equation

$$
z^{+}=D_{\theta} z+w, \quad y=C_{\theta} z+v
$$

where $w \in \mathbb{R}^{n}$ is a zero mean Gaussian white noise with covariance matrix $\Lambda, v \in \mathbb{R}^{p}$ is a zero mean Gaussian white noise with covariance matrix $\Omega, w, v$ mutually independent, $\mathbf{P}\left\{\left(D_{\theta}, C_{\theta}\right)=\left(D_{i}, C_{i}\right)\right\}=p$ for $i \neq 0$ and $p_{0}$ otherwise, iid. Let $\Xi=\operatorname{diag}\{\Lambda, \Omega\}$. Then for every $\beta \geq 0$, the following implication holds:

(i) If there exist matrices $\Phi_{0}=\left[\begin{array}{cl}\Phi_{\phi \phi} & 0 \\ 0 & 0\end{array}\right], \Phi_{i}=$ $\left[\begin{array}{cc}\Phi_{\phi \phi} & \Phi_{\phi y_{i}} \\ \Phi_{y_{i} \phi} & \Phi_{y_{i} y_{i}}\end{array}\right] \in \mathbb{R}^{(n+p) \times(n+p)}, i \in\{1, \ldots, \ell\}$ with $\Phi_{0}, \Phi_{i} \geq 0$ such that:

$$
\begin{gathered}
\Phi_{\phi \phi} \geq p_{0}\left[\begin{array}{l}
D_{0} \\
C_{0}
\end{array}\right] \Phi_{0}\left[\begin{array}{l}
D_{0} \\
C_{0}
\end{array}\right]^{T} \\
+p \sum_{i=0}^{\ell}\left\{\left[\begin{array}{l}
D_{i} \\
C_{i}
\end{array}\right] \Phi_{i}\left[\begin{array}{l}
D_{i} \\
C_{i}
\end{array}\right]^{T}\right\}+I \\
\beta \geq p_{0} \operatorname{tr}\left(\Phi_{0} \Xi\right)+p \sum_{i=1}^{\ell} \operatorname{tr}\left(\Phi_{i} \Xi\right)
\end{gathered}
$$

(ii) then there exists a map $\kappa:\{0,1, \ldots, \ell\} \times \mathbb{R}^{p} \rightarrow \mathbb{R}^{n}$ such that the state estimate $\hat{z}=\kappa(\theta, y)$ satisfies

$$
\mathbf{E}|z-\hat{z}|^{2} \leq \beta
$$

Moreover, if $K_{i}=\Phi_{\phi \phi}^{-1} \Phi_{\phi y_{i}}$ and $\left\{\Phi_{i}\right\}_{i=0}^{\ell}$ satisfy the conditions in (i), then (i) hold for the estimator

$$
\hat{z}^{+}=D_{\theta} \hat{z}+K_{\theta}\left(C_{\theta} z-y\right) .
$$

Proof: Let $K_{i}=\Phi_{\phi \phi}^{-1} \Phi_{\phi y_{i}}$. Let the evolution of $\hat{z}$ be governed by (21) and define $e=z-\hat{z}$. We have

$$
e^{+}=\left(D_{\theta}+K_{\theta} C_{\theta}\right) e+w+K_{\theta} v .
$$


Define $E_{i}=\mathbf{E}\left\{e e^{T} \mid \theta=i\right\}$. We have

$$
\begin{aligned}
E_{i} & =\mathbf{E}\left\{e^{+} e^{+^{T}} \mid \theta=i\right\} \\
& =\left[\begin{array}{ll}
I & K_{i}
\end{array}\right]\left(\left[\begin{array}{l}
D_{i} \\
C_{i}
\end{array}\right] E_{i}\left[\begin{array}{c}
D_{i} \\
C_{i}
\end{array}\right]^{T}+\Xi\right)\left[\begin{array}{ll}
I & K_{i}
\end{array}\right]^{T},
\end{aligned}
$$

and, thus

$$
\begin{aligned}
& \operatorname{tr}\left(\Phi_{\phi \phi} E_{i}\right) \\
& =\operatorname{tr}\left(\left[\begin{array}{ll}
I & K_{i}
\end{array}\right] \Phi_{\phi \phi}\left[\begin{array}{ll}
I & K_{i}
\end{array}\right]^{T}\left(\left[\begin{array}{l}
D_{i} \\
C_{i}
\end{array}\right] E_{i}\left[\begin{array}{l}
D_{i} \\
C_{i}
\end{array}\right]^{T}+\Xi\right)\right) \\
& \leq \operatorname{tr}\left(\Phi_{i}\left(\left[\begin{array}{l}
D_{i} \\
C_{i}
\end{array}\right] E_{i}\left[\begin{array}{l}
D_{i} \\
C_{i}
\end{array}\right]^{T}+\Xi\right)\right) \\
& =\operatorname{tr}\left(\left[\begin{array}{l}
D_{i} \\
C_{i}
\end{array}\right] \Phi_{i}\left[\begin{array}{l}
D_{i} \\
C_{i}
\end{array}\right]^{T} E_{i}+\Phi_{i} \Xi\right) \\
& =\operatorname{tr}\left(\left(\left[\begin{array}{l}
D_{i} \\
C_{i}
\end{array}\right] \Phi_{i}\left[\begin{array}{l}
D_{i} \\
C_{i}
\end{array}\right]^{T}+I\right) E_{i}-E_{i}+\Phi_{i} \Xi\right)
\end{aligned}
$$

by commutativity of the trace operator and the definition of $K_{i}$. Thus, by the law of total expectation,

$$
\begin{aligned}
& \operatorname{tr}\left(\Phi_{\phi \phi} \mathbf{E}\left\{e e^{T}\right\}\right)=\mathbf{E}\left\{\operatorname{tr}\left(\Phi_{i} \Xi\right)\right\}+ \\
& \mathbf{E}\left\{\operatorname{tr}\left(\left(\left[\begin{array}{l}
D_{i} \\
C_{i}
\end{array}\right] \Phi_{i}\left[\begin{array}{l}
D_{i} \\
C_{i}
\end{array}\right]^{T}+I\right) E_{i}\right)\right\}-\mathbf{E}\left\{E_{i}\right\},
\end{aligned}
$$

hence, rearranging,

$$
\begin{aligned}
\mathbf{E}\left\{E_{i}\right\} & =\mathbf{E}\left\{e e^{T}\right\} \\
\leq & \mathbf{E}\left\{\operatorname{tr}\left(\left(\left[\begin{array}{c}
D_{i} \\
C_{i}
\end{array}\right] \Phi_{i}\left[\begin{array}{c}
D_{i} \\
C_{i}
\end{array}\right]^{T}+I\right) E_{i}\right)\right\} \\
& \quad-\operatorname{tr}\left(\Phi_{\phi \phi} \mathbf{E}\left\{e e^{T}\right\}\right)+\beta \\
\leq & \operatorname{tr}\left(\Phi_{\phi \phi} \mathbf{E}\left\{e e^{T}\right\}\right)-\operatorname{tr}\left(\Phi_{\phi \phi} \mathbf{E}\left\{e e^{T}\right\}\right)+\beta=\beta .
\end{aligned}
$$

The following theorem develops sufficient conditions for the existence of a stabilizing control law for the case where outputs are scheduled. Essentially, it is separation principle for controller design for randomly switched systems of the form (15)-(16).

Theorem 3: Let $A_{\theta}, B_{\theta}, C_{\theta}$ denote the system matrices for (15)-(16). Define the feedback law $u$ by

(a)

$$
\begin{aligned}
\hat{z}^{+} & =\left(A_{\theta}+B_{\theta} L_{\theta}\right) \hat{z}+K_{\theta}\left(C_{\theta} \hat{z}-y\right) \\
u & =L_{\theta} \hat{z},
\end{aligned}
$$

(b) where $L_{\theta}$ and $K_{\theta}$ were defined Theorem 1 and Theorem 2 (for system (15)-(16)), respectively; and,

(c) where $\left\{X_{i}\right\}_{i=0}^{\ell}>0$ in Theorem 1 and $\left\{\Phi_{i}\right\}_{i=0}^{\ell}>0$ in Theorem 2 satisfy the conditions of the respective theorems with the minimum feasible $\gamma$ and $\beta .^{2}$

Then $u$ satisfies $\mathbf{E}|C z+D u|^{2} \leq \gamma$.

Proof: The proof follows from combining Theorem 1 and Theorem 2 and noting that the state estimate error is bounded, stationary and uncorrelated with $z$.

\footnotetext{
${ }^{2}$ We require strict feasibility of the respective LMIs, hence, the respective matrices are strictly positive definite.
}

\section{CASe Study}

We consider the networked control of a $\mathrm{CH}-47$ tandemrotor helicopter in horizontal motion about a nominal airspeed of $40 \mathrm{knots} / \mathrm{hr}$ as discussed in [13]:

$$
\dot{x}=A x+B u,
$$

where

$$
F=\left[\begin{array}{cccc}
-0.02 & 0.005 & 2.4 & -32 \\
-0.14 & 0.44 & -1.3 & -30 \\
0 & 0.018 & -1.6 & 1.2 \\
0 & 0 & 1 & 0
\end{array}\right] G=\left[\begin{array}{cc}
0.14 & -0.12 \\
0.36 & -8.6 \\
0.35 & 0.009 \\
0 & 0
\end{array}\right]
$$

The inputs $u_{1}, u_{2}$ are collective rotor thrust and differential collective rotor thrust respectively. We assume that only inputs are transmitted via the network $(\ell=2)$ and seek an optimal feedback controller design. We set $s=0.2$ seconds, $p_{0}=0.2$ and, hence, the set of system matrices $(i=0,1,2)$ are given by

$$
\begin{aligned}
& {\left[\begin{array}{cccccc}
1.4993 & 0.8510 & 1.0857 & 1.7557 & 0.6322 & -0.3560 \\
-0.0932 & 0.4290 & -0.0403 & 0.0083 & 0.7705 & 0.0092
\end{array}\right]} \\
& A_{0}=\mid \begin{array}{cccccc}
-0.0932 & 0.4290 & -0.0403 & 0.0083 & 0.7705 & 0.0092 \\
0.1543 & 0.5827 & 0.4086 & 0.6890 & 0.5996 & -0.3839 \\
0.0794 & 0.5590 & 0.1656 & 0.8321 & 0.5842 & -0.0600
\end{array} \\
& {\left[\begin{array}{ccccccc}
0.0794 & 0.5590 & 0.1656 & 0.8321 & 0.5842 & -0.0600 \\
0 & 0 & 0 & 0 & 1.0000 & 0 \\
0 & 0 & 0 & 0 & 0 & 1.0000
\end{array}\right.} \\
& A_{1}=\left[\begin{array}{cccccc}
1.4993 & 0.8510 & 1.0857 & 1.7557 & 0.6322 & -0.3560 \\
-0.0932 & 0.4290 & -0.0403 & 0.0083 & 0.7705 & 0.0092 \\
0.1543 & 0.5827 & 0.4086 & 0.6890 & 0.5996 & -0.3839 \\
0.0794 & 0.5590 & 0.1656 & 0.8321 & 0.5842 & -0.0600 \\
0 & 0 & 0 & 0 & 0 & 0 \\
0 & 0 & 0 & 0 & 0 & 1.0000
\end{array}\right] \\
& A_{2}=\left[\begin{array}{cccccc}
1.4993 & 0.8510 & 1.0857 & 1.7557 & 0.6322 & -0.3560 \\
-0.0932 & 0.4290 & -0.0403 & 0.0083 & 0.7705 & 0.0092 \\
0.1543 & 0.5827 & 0.4086 & 0.6890 & 0.5996 & -0.3839 \\
0.0794 & 0.5590 & 0.1656 & 0.8321 & 0.5842 & -0.0600 \\
0 & 0 & 0 & 0 & 1.0000 & 0 \\
0 & 0 & 0 & 0 & 0 & 0
\end{array}\right] \\
& B_{0}=0, \quad B_{1}=\left[\begin{array}{cc}
0 & 0 \\
5.6790 & 0 \\
1.1360 & 0 \\
1.1360 & 0 \\
1.0000 & 0 \\
0 & 0
\end{array}\right] \quad B_{2}=\left[\begin{array}{cc}
0 & 0 \\
0 & 0 \\
0 & -3.1460 \\
0 & 0 \\
0 & 0 \\
0 & 1.0000
\end{array}\right] \text {. }
\end{aligned}
$$

Let the $\mathbf{E}\left\{w w^{T}\right\}=I$ and, with respect to Theorem 1, let $C=I, D=I$. The respective LMI (13) is indeed feasible and the optimal control law is given by $u_{i}=L_{i} z, i=\{1,2\}$, where

$$
\begin{aligned}
L_{1} & =\left[\begin{array}{cccccc}
-0.090 & -0.196 & -0.099 & -0.243 & -0.224 & 0.094 \\
0 & 0 & 0 & 0 & 0 & 0
\end{array}\right] \\
L_{2} & =\left[\begin{array}{cccccc}
0 & 0 & 0 & 0 & 0 & 0 \\
0.4092 & 0.8365 & 0.4420 & 1.0635 & 1.5808 & -0.2128
\end{array}\right] .
\end{aligned}
$$

Note that, as expected, the feedback matrix is identically zero for the column corresponding to the input that is not being transmitted. Moreover, $A_{0}$ is unstable and $A_{1}+B_{1} L_{1}$, $A_{2}+B_{2} L_{2}$ each have a single eigenvalue on the unit circle (all others are in the interior of the unit circle) thus, no single mode of the jump system is stable itself.

We have $\operatorname{tr}\left(X_{z z}\right)+\operatorname{tr}\left(X_{u_{1} u_{1}}\right)+\operatorname{tr}\left(X_{u_{2} u_{2}}\right)=615.36$ and, in particular, $\operatorname{tr}\left(X_{z z}\right)=584.97$, thus, the resultant system is mean-square stable.

Finally, the batch reactor system is simulated with the optimal feedback gains $L_{1}, L_{2}$. To that end, we set $z_{0}=$ $(0, \ldots, 0)^{T}$ and simulated the system over $n=64800000$ 
iterations, corresponding to 150 days of simulated operation. The ensemble average state norm is given by $1 / n \sum_{i=1}^{n} z(i) z(i)^{T}=560.3963$, corresponding well with the theoretical value of 584.97

\section{CONCLUSION}

This paper described a framework for modeling linear networked control systems using Ethernet-like networks with random transmission, packet dropouts and random intertransmission times. We provided an optimal controller design framework for the case where only inputs are scheduled for transmissions and full state measurements are available. The optimal solution was described using dynamic programming for the finite-horizon problem and, restricting to the case where inter-transmission intervals are equidistant and deterministic, we provided tractable necessary and sufficient LMI conditions for existence of the infinite-horizon problem as well as the associated optimal feedback control.

This paper also tackled the optimal design problem for NCS where outputs are scheduled for transmission. In this case, we immediately restricted out attention to intertransmission intervals that are equidistant and deterministic and provided sufficient conditions in the form of LMIs for the existence of the solution of the infinite-horizon design problem and the associated optimal control. As in the classical network-free case, the optimal solution is a combination of an optimal state estimator together with an optimal state feedback controller.

Several key issues remain in the form of asserting that the output feedback LMI conditions are necessary and not merely sufficient, developing tractable solutions for random inter-transmission intervals and allowing both outputs and inputs to be randomly scheduled simultaneously.

\section{REFERENCES}

[1] M. Tabbara, D. Nešić, and A. Teel, "Stability of wireless and wireline networked control systems," to appear in IEEE Trans. Automat. Contr., 2007.

[2] M. Tabbara and D. Nešić, "Input-Output Stability of Networked Control Systems with Stochastic Protocols and Channels," to appear in IEEE Trans. Automat. Contr., 2007.

[3] D. Nešić and A. Teel, "Input-output stability properties of networked control systems," IEEE Trans. Automat. Contr., vol. 49, no. 10, pp. 1650-1667, 2004.

[4] _ " "Input-to-state stability of networked control system," Automatica, vol. 40, no. 12, pp. 2121-2128, 2004.

[5] G. Nair and R. Evans, "Stabilizability of stochastic linear systems with finite feedback data rates," SIAM J. Contr. Optimization, vol. 43, no. 2, pp. 413-436, July 2004.

[6] J. Braslavsky, R. Middleton, and J. Freudenberg, "Feedback stabilization over signal-to-noise ratio constrained channels," in American Control Conference, vol. 6, 2004, pp. 4903-4908.

[7] D. Dačić and D. Nešić, "Simultaneous controller and protocol design for networked control systems with packet based communication," in Proc. Conf. Decis. Contr., San Diego, CA, Dec. 2006, pp. 508-513.

[8] A. S. Tanenbaum, Computer Networks, 4th ed. Prentice Hall, 2003.

[9] N. Dunford and J. T. Schwartz, Linear Operators I, General Theory. Interscience, 1963.

[10] D. Bertsekas, Dynamic Programming and Optimal Control, 2nd ed. Athena Scientific, 2000, vol. 1.

[11] O. Costa and M. Fragoso, "Discrete-Time LQ-Optimal Control Problems for Infinite Markov Jump Parameter Systems," IEEE Trans. Automat. Contr., vol. 40, no. 12, pp. 2076-2088, 1995.
[12] A. Rantzer, "A separation principle for distributed control," in Proc. Conf. Decis. Contr., San Diego, CA, 2006, pp. 3609-3613.

[13] J. Doyle and G. Stein, "Mutlivariable feedback design: Concepts for a classic/modern synthesis," IEEE Trans. Automat. Contr., vol. 26, no. 1, pp. 4-16, 1981. 